\title{
A compositionality machine realized by a hierarchic architecture of synfire chains
}

\author{
Sven Schrader ${ }^{*}$, Markus Diesmann ${ }^{2,3,4}$ and Abigail Morrison ${ }^{5,4,2}$ \\ Honda Research Institute, Offenbach, Germany \\ 2 RIKEN Brain Science Institute, Wako City, Japan \\ ${ }^{3}$ RIKEN Computational Science Research Program, Wako City, Japan \\ ${ }^{4}$ Bernstein Center Freiburg, Albert-Ludwigs University, Freiburg, Germany \\ ${ }^{5}$ Functional Neural Circuits Group, Faculty of Biology, Albert-Ludwigs University, Freiburg, Germany
}

\section{Edited by:}

Israel Nelken, Hebrew University, Israel

\section{Reviewed by:}

John A. Hertz, Niels Bohr Institute, Denmark

Peter Latham, University College

London, UK

\section{*Correspondence:}

Sven Schrader, Honda Research Institute Europe GmbH, Carl-LegienStr. 30, 63073 Offenbach am Main, Germany.

e-mail:sven.schrader@honda-ri.de
The composition of complex behavior is thought to rely on the concurrent and sequential activation of simpler action components, or primitives. Systems of synfire chains have previously been proposed to account for either the simultaneous or the sequential aspects of compositionality; however, the compatibility of the two aspects has so far not been addressed. Moreover, the simultaneous activation of primitives has up until now only been investigated in the context of reactive computations, i.e., the perception of stimuli. In this study we demonstrate how a hierarchical organization of synfire chains is capable of generating both aspects of compositionality for proactive computations such as the generation of complex and ongoing action. To this end, we develop a network model consisting of two layers of synfire chains. Using simple drawing strokes as a visualization of abstract primitives, we map the feed-forward activity of the upper level synfire chains to motion in two-dimensional space. Our model is capable of producing drawing strokes that are combinations of primitive strokes by binding together the corresponding chains. Moreover, when the lower layer of the network is constructed in a closed-loop fashion, drawing strokes are generated sequentially. The generated pattern can be random or deterministic, depending on the connection pattern between the lower level chains. We propose quantitative measures for simultaneity and sequentiality, revealing a wide parameter range in which both aspects are fulfilled. Finally, we investigate the spiking activity of our model to propose candidate signatures of synfire chain computation in measurements of neural activity during action execution.

Keywords: compositionality, synfire chains, movement primitives, simulation

\section{INTRODUCTION}

How is a complex object perceived when it is encountered for the first time? Although it is possible that the brain develops new categories for each new object, it is more likely that it breaks the complex object down into simpler objects and their component features such as shape and color. Thus, when a new object is encountered, it is analyzed according to its primitives and the collection of active primitives forms the complex object (e.g., Tanaka, 2003). The concept of creating complex objects out of the sum of their parts is known as compositionality.

The principle of compositionality as stated above is not restricted to reactive computations such as the comprehension of perceptions, it can also be applied to proactive computations, for example planning and executing complex motor programs on the basis of an underlying hierarchy of motion primitives. Evidence for the existence of movement primitives has been found in several studies, either on the actuator/kinematic/dynamic or on the neural level. Direction specificity of neural coding has been demonstrated by Georgopoulos et al. (1982) and can be regarded as the most elementary neural realization of primitives. Mussa-Ivaldi et al. (1994) showed that spinal stimulation of a frog did not produce uniform leg movement. Instead, the stimulated area represented a static "force field" acting on the leg, dragging the foot to a fixed position. Surprisingly, two force fields superimpose linearly when stimulated together. Furthermore, if an internal model is assumed, human reaching movements can be separated into primitives showing Gaussian (Thoroughman and Shadmehr, 2000) or log-Gaussian (Plamondon, 1995) speed profiles. Likewise, random scribbling from monkeys can be broken into unit segments (Soechting and Terzuolo, 1987) and appears to be composed from a small number of parabolas (Polyakov et al., 2009a,b). The presence of motor primitives is especially prominent during learning of new movements (Sosnik et al., 2004) or recovery from stroke (Rohrer et al., 2002).

The above studies reveal two aspects to the compositionality of complex behavior. The first aspect is that primitives provide a basic set of behaviors whose simultaneous combination yield the final action. We refer to this aspect as simultaneity. Secondly, primitives can be arranged in a series. We will refer to this as sequentiality. Whereas some compositional systems only require one of these computational features, others, most notably motor control, require both.

The question of how such compositional systems are realized in the neural substrate is an unresolved issue. Undoubtedly, aspects of movement can be inferred on the basis of firing rates (e.g., Georgopoulos et al., 1982). An alternative proposition is that information is encoded in the precise time of occurrence of an 
action potential. A number of studies have revealed the occurrence of precise spike timing (Eckhorn et al., 1988; Gray and Singer, 1989; Abeles et al., 1993; Prut et al., 1998; Pulvermüller and Shtyrov, 2009) with respect to critical features of experimental protocols, which strongly suggests temporal coding. These strategies are not mutually exclusive; Masuda (2006) demonstrated how the two formulations can coexist.

The mechanism behind the formation of such fine temporal structure is still unknown. A single post-synaptic potential (PSP) is relatively small compared to the range of membrane potential fluctuations and therefore not very likely to elicit a spike (Abeles, 1982; Matsumura et al., 1996; Hasenstaub et al., 2005). However, reliable post-synaptic action potential generation can be triggered by a near-synchronous pulse of a pre-synaptic population ("just-enough, just-in-time"; Douglas and Martin, 2007; Anderson and Martin, 2009). If applied inductively, this insight leads to the concept of a volley of synchronous activity propagating along a structure known as a synfire chain (Abeles, 1991). In its simplest formulation, a synfire chain is a feed-forward subnetwork consisting of a row of neuron groups in which each neuron in a group projects to the neurons in the following group (see Figure 1). The propagation of pulse packets in synfire chains has been shown to be stable under quite general conditions (Diesmann et al., 1999; Gewaltig et al., 2001).

Although the synfire concept was originally developed to account for precise firing patterns in the brain, there is as yet no unequivocal proof that they exist. The main evidence in favor of the hypothesis is that the repeating spatiotemporal firing patterns that a synfire chain would generate can be measured during experiments (Abeles et al., 1993; Prut et al., 1998; Baker et al., 2001; Kohn and Smith, 2005; Shmiel et al., 2005, 2006; Long et al., 2010), and have even been shown to re-occur after several minutes (Ikegaya et al., 2004). In awake animals precise firing patterns are related to behavior, but are usually not tightly time locked to any known external event. Other studies have used surrogate or shuffled spike data to challenge these results, suggesting that such patterns occur by chance rather than as a result of an underlying feed-forward structure (Oram et al., 1999; Baker and Lemon, 2000; Mokeichev et al., 2007). These studies have in turn been criticized on the basis of the underlying null hypothesis (Date et al., 1999; Abeles and Gat, 2001) or the nature of the surrogate data. Abeles and Gat (2001) and Gerstein (2004) argue that the surrogates used to challenge the synfire hypothesis introduced excess structure into the artificial spike trains, whereas Ikegaya et al. (2008) contends that the detection algorithm used in the respective earlier studies was not sufficiently sensitive. Contributing to the ongoing debate on the existence of precise firing or feed-forward networks is outside the scope of the current manuscript. In the following, we therefore assume their existence as a useful working hypothesis.

Bienenstock (1995) conjectured that perception or recall of entities and actions or relations between them are based on the co-operative action of many synfire chains. In this hypothesis, each primitive is realized by a synfire chain, and a specific object can be represented by a particular composition of the underlying primitive chains. This requires some degree of interaction between synfire chains in order to determine which primitives are involved in the composition. Bienenstock (1995) speculated that this interaction is mediated by weak inter-connections between the chains, as illustrated in Figures 2A,B. Thus, if stimulated at approximately the same time, chains can aggregate their traveling waves to form one front (see Figure 2C). This mechanism is referred to as synfire binding. It has been demonstrated that synfire binding can indeed occur (Arnoldi and Brauer, 1996) and that it is a feasible mechanism to compose complex objects out of multiple perceptual primitives (Abeles et al., 2004). Those networks can reliably discriminate between different triple-pulses. Unlike attractor networks, the resultant network states are transiently active followed by a return to steady low-rate background activity.

The sequential aspect of compositionality has also been attributed to activity in an appropriate architecture of synfire chains. The concatenation of syllables in birdsong has been proposed to result from the consecutive activation of synfire chains, each representing one syllable (Hahnloser et al., 2002; Li and Greenside, 2006; Kozhevnikov and Fee, 2007; Jin et al., 2007; Jin, 2009; Hanuschkin et al., 2010a). Thus, a bird song is realized by a particular sequence of primitives. A song syntax can be realized by the connectivity between the chains representing individual syllables: if syllable A can be followed by a repetition of $\mathrm{A}$ or by syllable $\mathrm{B}$, the final group
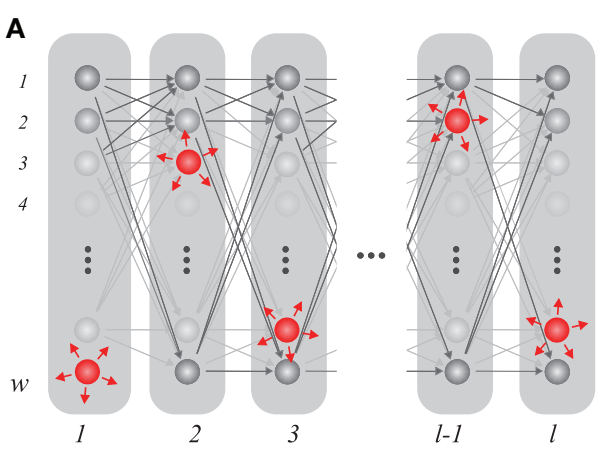

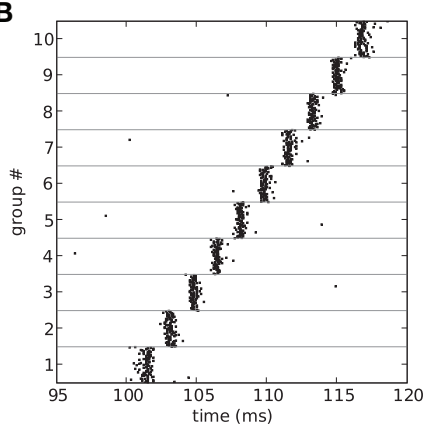

FIGURE 1 | A simple synfire chain. (A) Wiring diagram of a synfire chain. Excitatory neurons in each group (gray discs) have feed-forward connections to all neurons in the next group (gray arrows); in this version of the synfire chain model, inhibitory neurons in each group (red discs) project to random neurons in the entire network to create a global inhibition. (B) Synfire activity. If the first group is sufficiently stimulated, a volley of activity propagates through the chain. Each dot represents the spike time of a neuron; horizontal lines separate the spikes of consecutive neuron groups. 

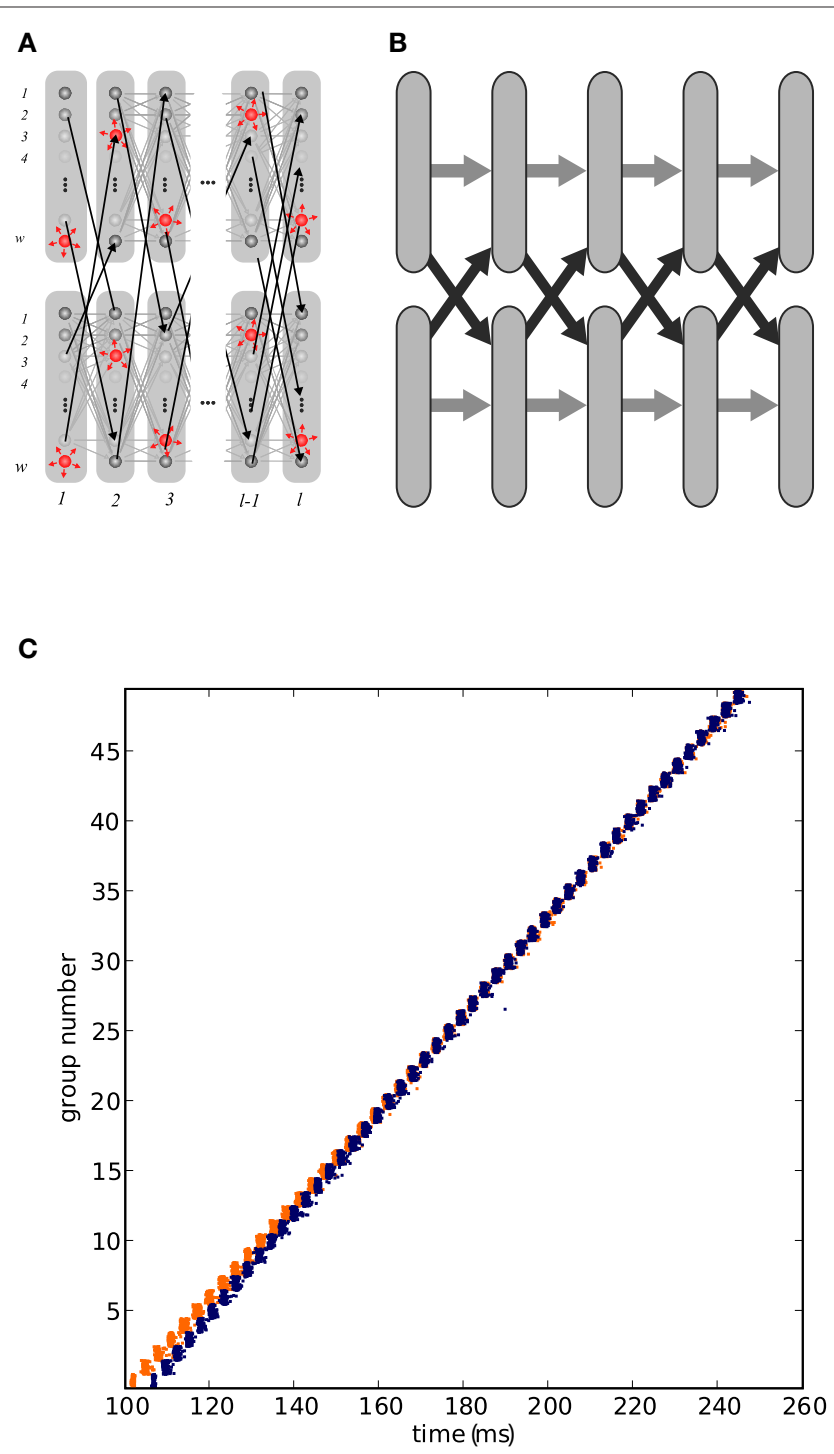

FIGURE 2 | Interacting synfire chains. (A) Wiring diagram of two cross-connected chains. Each chain has internal connections as illustrated in Figure 1A; the two chains are connected via a few excitatory synapses (black arrows) which connect a given group of one chain to the next group in the parallel chain. (B) Schematic view of the synfire network in (A). Gray bars denote synfire groups, gray arrows the feed forward connections and black arrows the interconnections. (C) Interacting activity: if the first groups of the chain are stimulated nearly synchronously, the wave front of the second chain (blue) catches up with that of the first chain (orange) to form a unified wave front.

of the synfire chain representing $A$ is connected in a feed-forward fashion to the initial groups of chains A and B. Similarly, a network of interconnected synfire chains can account for key experimental findings in free monkey scribbling with respect to cortical activity and the statistics of the generated trajectories (Hanuschkin et al., 2010b).

So far, modeling studies have investigated the simultaneous and sequential aspects of compositionality in isolation. However, it has yet to be shown that synfire chains can provide the substrate for compositional systems requiring both computational features, such as motor control. It is not clear that the aspects can be combined, as the models described above that exhibit simultaneity or sequentiality rely on entirely different mechanisms: simultaneity is realized by synfire chain binding, whereas sequentiality is realized by the propagation of activity from the end of one chain to the beginning of the other. It is therefore not obvious that the computational features are compatible either in terms of network architecture or in terms of parameters. Here, we demonstrate that both types of computation can indeed be carried out in a single network model. The resulting behavior exhibits both simultaneous and sequential combination of primitives and is considerably richer than that generated by previous models based on synfire chains. The discovery that the two types of computation are complementary provides support for the hypothesis that synfire chains constitute a key element of neural computation.

In Sec. 3.1 we develop a two-layer network of synfire chains. To illustrate the computational features of our model, we map the feed-forward activity in each of the upper level chains to simple drawing strokes in four different directions. We first show that synfire chain binding allows simultaneous execution of primitives, resulting in composite drawing strokes. Secondly, in Sec. 3.2 we demonstrate that if the lower level chains are constructed in a closed-loop manner, the motion is no longer bounded by synfire chain termination. In this case, the model generates an ongoing sequence of individual and composite drawing strokes. The patterns generated by the network can be either random or deterministic, depending on the connectivity at the seam of the lower level loops. In Sec. 3.3 we investigate the behavior of the network as a function of the inhibition in the upper and lover levels and determine a range of parameters for which the criteria of simultaneity and sequentiality are satisfactorily fulfilled. Finally, in Sec. 3.4 we investigate the spiking activity of our model during the generation of complex behavior and propose candidate signatures for synfire chain activity in massively parallel recordings of spike trains and mass signals.

Preliminary results have been published in abstract form (Schrader et al., 2007).

\section{MATERIALS AND METHODS \\ 2.1 SYNFIRE BINDING AND COMPETITION}

As demonstrated by Arnoldi and Brauer (1996), synfire binding occurs when two or more cross-connected synfire chains synchronize their traveling pulses after a near-synchronous stimulation (see Figure 2). If the two chains are stimulated within a sufficiently short interval, the chain which is stimulated later (the lagging chain) speeds up its propagation of pulses until the activity is synchronized in corresponding groups of the chains. The binding mechanism can be fully described by a wave theoretical approach (Hayon et al., 2005). The detection of the binding state can be realized by a third synfire chain, as is demonstrated in Figure 3A. The upper level chain can only be activated if the activity of the lower level chains is sufficiently synchronized. Figure $3 \mathbf{B}$ shows the activity in the chains associated with the detection of binding states. At $100 \mathrm{~ms}$ the inter-stimulus interval is only $10 \mathrm{~ms}$, so the two lower level chains bind and activate the upper level chain. The inter-stimulus interval of $30 \mathrm{~ms}$ at $400 \mathrm{~ms}$ is too great; no binding takes place and the increase in global inhibition due to both lower level chains being active even causes one of the chains to fail in this example. Abeles et al. (2004) showed that this basic hierarchy of chains is sufficient to 
A

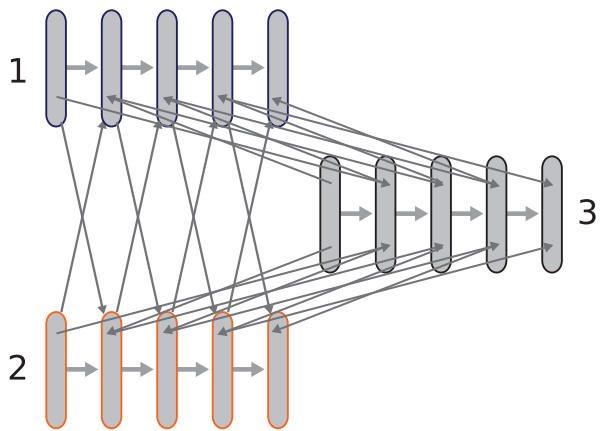

C

1

3

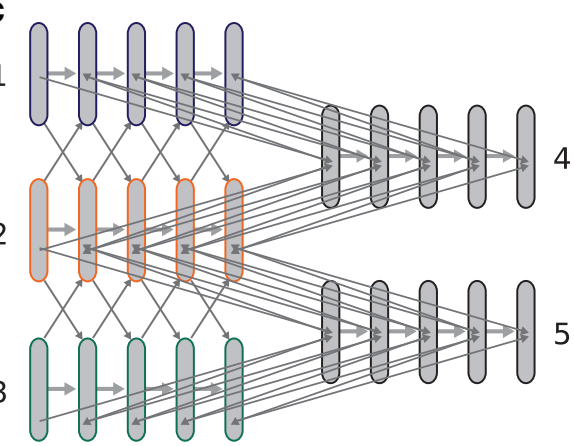

B

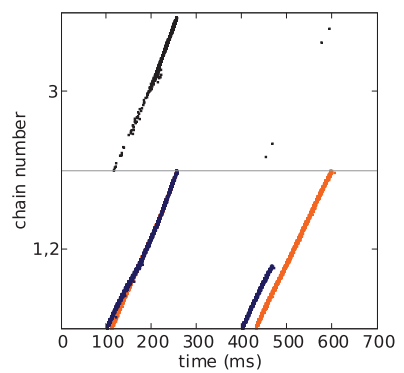

D

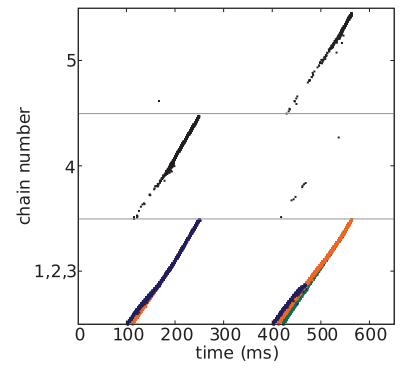

FIGURE 3 | Detection and discrimination of binding states in networks of synfire chains. (A) Simple three-chain network. Each pair of chains $(1,2),(1,3)$, and $(2,3)$ are interconnected as shown in Figure 2A. The upper level chain $(3$, black) serves as a readout chain to detect the binding of the lower level chains (1, blue; 2 , orange). (B) Detection of binding in the three-chain network in (A), coloring of spike time markers in the lower panel indicates chain membership of neuron. The two lower level chains are stimulated at $t_{1}=100$ and $t_{2}=110 \mathrm{~ms}$. The chains bind and activate the third chain. The lower level chains are stimulated again at $t_{1}=400$ and $t_{2}=430 \mathrm{~ms}$. In this case, the chains do not bind as the stimulus interval of $30 \mathrm{~ms}$ is too large. (C) Five-chain network. Here, three chains (1, blue; 2 , orange; 3 , green) constitute the lower level chains and two upper level chains (4-5, black) serve as readout chains. Interconnections are indicated by gray arrows. (D) As in (B), but for the five-chain network shown in (C). Chains 1 and 2 are stimulated at $t_{1}=100$ and $t_{2}=115 \mathrm{~ms}$. They bind together and activate the upper level chain 4 . All three lower level chains are stimulated again at $t_{1}=400, t_{2}=415$, and $t_{3}=420 \mathrm{~ms}$. In this case chains 2 and 3 bind, as they have the smallest inter-stimulus interval. Their binding causes the activation of upper level chain 5. model compositionality: the lower level chains represent primitive features and the upper level chain represents the composite object. The representation of the composite object is only active if the component features are detected near-synchronously.

In addition to binding primitive features, a second property necessary for the computation of compositionality is competition. To prevent the representation of one nonsensical "mega object," which corresponds in the network model to too many chains binding together, only the most relevant detected features should be bound. In a network of chains, this can be easily realized by the presence of inhibitory neurons. In the small networks studied here, it is sufficient to include inhibitory neurons in the feed-forward structures that project non-specifically to other neurons in the same layer of the hierarchical network (see Figure 1A). When chains bind together, the resultant synchronous inhibition prevents further chains from joining the binding state. The activity in a fivechain network (Figure 3C) associated with competition between binding states is shown in Figure 3D. At around $100 \mathrm{~ms}$ the lower level chains 1 and 2 are stimulated with an inter-stimulus interval of $15 \mathrm{~ms}$, which is short enough to allow the chains to bind and thus activate the upper level chain 4 . At around $400 \mathrm{~ms}$ chains 1 and 2 are once again stimulated with an inter-stimulus interval of $15 \mathrm{~ms}$, however the shorter inter-stimulus interval of $5 \mathrm{~ms}$ between chains 2 and 3 allows those two to bind instead. The synchronous inhibition in chains 2 and 3 prevents chain 1 from joining the binding state. Consequently, only the upper level chain 5 is activated. This dynamics is equivalent to a competition between possible bindings which is biased toward those subsets of features that occur with a higher degree of synchrony; a bias toward binding subsets of features that are detected earlier has also previously been demonstrated (Abeles et al., 2004).

In the current paper, we assume homogeneous layer-specific inhibition, with synaptic strength empirically adjusted such that at most two upper level chains can be simultaneously active (see Table 2). For more complex networks, the specificity and heterogeneity of inhibitory projections are likely to be crucial factors affecting the network's computational properties.

\subsection{MAPPING ACTIVITY TO ACTIONS}

Abeles et al. (2004) demonstrated that a hierarchical network of synfire chains can detect near-synchronous stimuli and discriminate between different subsets, i.e., that it can compute compositionality of perception. Therefore the purpose of the upper level chains was simply to act as binary indicators of lower level binding states. 
The feed-forward dynamics of the response to lower level binding was not further considered. Here, we focus on the ability of such networks to generate actions. In particular, we want to illustrate the capabilities of a synfire chain network to generate both the sequential and simultaneous aspects of compositionality. To this end, we choose a task that is on the one hand as simple as possible (in terms of the number of primitives) and on the other hand easy to visualize. We map the activity of four upper level chains to simple drawing strokes. The mapping of activity to drawing actions facilitates particularly easy visualization of the computational features of the network, i.e., it is immediately apparent to the eye when two strokes have been superimposed or when one follows another. However, the choice of drawing strokes is not intended to suggest an account of movement generation, which would require a much more sophisticated model. Indeed, any set of combinable actions could have been chosen.

To generate the drawing strokes, we consider the neurons in a given upper level chain $j$ to have a preferred direction, characterized by its unit velocity $\mathbf{p}_{j}$ (see Georgopoulos et al., 1982). The net drawing velocity generated by a network is determined by summing over the activity of all the upper level chains:

$$
\dot{\mathbf{x}}(t)=\sum_{j} \sum_{k} w_{k} a_{j}^{k}(t) \mathbf{p}_{j}
$$

where $a_{j}^{k}(t)$ is the activity of the $k$ th group in the $j$ th chain as expressed by a population histogram with a bin size of $h=5 \mathrm{~ms}$ and $w_{k}$ is the weight of the contribution of the $k$ th group in a chain. This bin size is the finest sampling interval possible without resolving the individual contributions of the synfire groups. For this study, we consider only a uniform velocity profile, $w_{k}=w$. The movement trajectory is then obtained by integrating the population vector $\dot{\mathbf{x}}(t)$ using the initial condition $\mathbf{x}(0)=(0,0)$ for each trial. No further smoothing of the neuronal activity or trajectory is carried out.

\subsection{MEASURES FOR SIMULTANEITY AND SEQUENTIALITY}

We define two measures to quantify the extent to which the network model realizes the simultaneous and sequential aspects of compositionality. In the full model, activation occurs in rounds due to the cyclical architecture of the lower level chains. In each round of activation either 0 , one, or more than one upper level chain is active.

First, we consider the activity $a_{j, i}$ of a single upper level chain $j$, where $i$ indicates the round of activation. We set $a_{j, i}$ to 0 if the chain is not actively propagating a volley of action potentials (i.e., it responds with fewer than 2 spikes per synfire group). We define the relative contribution $a_{j, i}^{\prime}=a_{j, i} / \Sigma_{x} a_{x, i}$ of a chain $j$ to the overall chain activity during round $i$. Note that, due to variable propagation times, $i$ does not correspond to absolute time. The measure of simultaneity for network activity is then defined as the average maximal activities over all rounds,

$$
p_{\text {sim }}=\frac{1}{N} \sum_{i=1}^{N} \max \left(a_{j, i}^{\prime}\right),
$$

where $N$ is the total number of rounds in which at least one upper level chain was active, i.e., $a_{j, i}>0$ for at least one $j$. We further define the measure of sequentiality $p_{\text {seq }}$ to be the proportion of rounds of activation in which at least one upper level chain is active.

\subsection{NUMERICAL SIMULATIONS}

We performed numerical simulations of networks of synfire chains. Simulations were performed with NEST revision 8733 (see www. nest-initiative.org and Gewaltig and Diesmann, 2007) using a computational step size of $0.1 \mathrm{~ms}$. This software is capable of simulating large-scale networks by means of distributed computing (Morrison et al., 2005). All simulations were performed on an AMD Opteron Dual Core SMP machine with four processors running Linux. To allow other researchers to perform their own experiments, at the time of publication we are making a module available for download at the NEST Initiative website containing all relevant scripts.

\subsubsection{Neuron model}

The model used in this study is a current-based leaky integrate-andfire (IAF) point neuron (Tuckwell, 1988). Its membrane potential $V$ is described by a linear differential equation

$$
\frac{d V}{d t}=-\frac{1}{\tau_{\mathrm{m}}} V+\frac{1}{C_{\mathrm{m}}} I_{\text {syn }}(t)
$$

where $\tau_{\mathrm{m}}$ is the membrane time constant, $C_{\mathrm{m}}$ is the membrane capacitance and $I_{\text {syn }}$ the synaptic current. If the membrane potential exceeds a fixed threshold $\theta$, a spike is emitted and the potential is immediately set to its reset value $V_{\text {reset }}$. During the subsequent refractory period $\tau_{\text {ref }}$ the membrane potential is clamped at $V_{\text {reset }}$. A single post-synaptic current (PSC) is modeled as an $\alpha$-function (Jack et al., 1983):

$$
I_{\alpha}(t)=\frac{e}{\tau_{\alpha}} t e^{-t / \tau_{\alpha}}
$$

where $\tau_{\alpha}$ is the rise time. The total synaptic current $I_{\text {syn }}$ is then

$$
I_{\text {syn }}=\sum_{i} \sum_{s \in S_{i}} J_{i} I_{\alpha}(t-s-d)
$$

where $i$ runs over all pre-synaptic neurons and $s$ over the set of all spike times $S_{i}$ of a pre-synaptic neuron $i$. $J$ is the peak value of the PSC and $d$ the synaptic delay. As the passive membrane potential of the integrate-and-fire neuron is a linear system, Eq. 3 can be solved numerically without approximation (Rotter and Diesmann, 1999; Plesser and Diesmann, 2009). The neuronal parameters used in this study are given in Table 2 .

\section{RESULTS}

\subsection{SIMULTANEOUS COMPOSITION OF PRIMITIVES}

In this section we show that the binding and competition properties exhibited by hierarchic networks of synfire chains (Sec. 2.1) are sufficient to enable the composition of actions from simpler actions. We construct a two layer network of synfire chains, illustrated in Figure 4A. Each synfire chain is internally connected according to the feedforward connectivity described in Abeles et al. (2004) and illustrated in Figure 2A, i.e., containing both excitatory neurons (100) which constitute the feed-forward connections and globally projecting inhibitory neurons (25) to introduce a competition between possible bindings. Unlike the five chain network shown in Figure 3C, in our model the strength of the inhibition is adjusted to allow as many as three lower level chains to simultaneously enter a binding state. Each upper level chain is activated by the binding of the two specific 

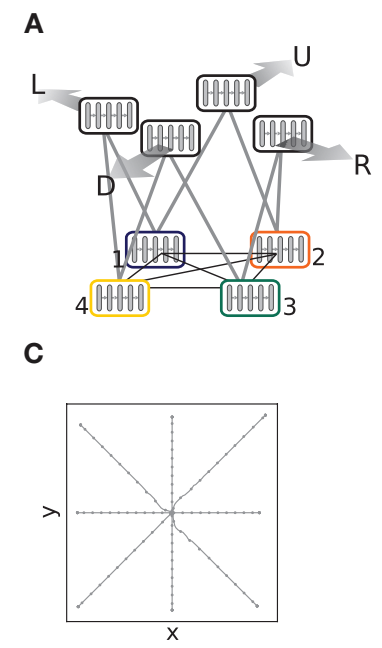

B

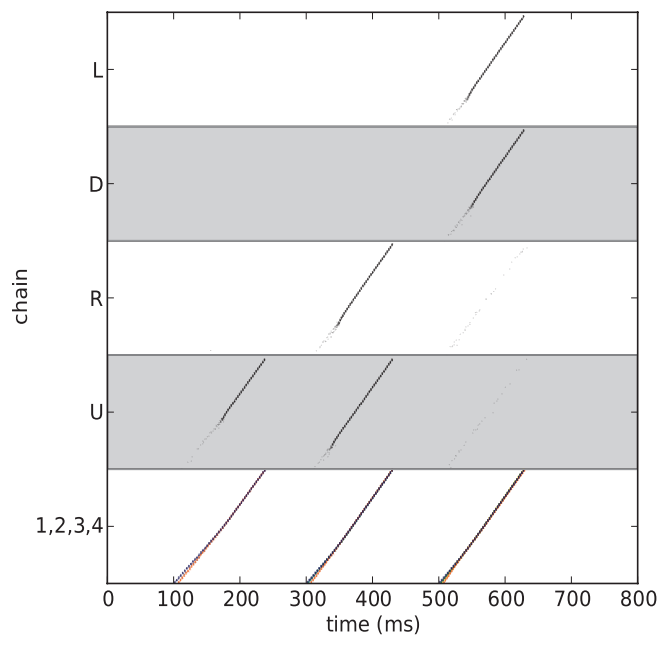

FIGURE 4 | A composition machine for simple drawing strokes. (A) Synfire chain connectivity. Lower level chains (1-4) are connected all-to-all to allow any possible binding. Cross-connections are as described in Figure 2A. Upper level chains (named "left," "up," "down," and "right") are interconnected with specific lower level chains as indicated by the gray arrows. (B) Neuronal activity during binding. Depending on the inter-stimulus interval of the lower level chains, specific upper level chains or combinations of upper level chains are activated (see text for details). (C) The eight different drawing strokes generated by the synfire network shown in (A). The directions up, left, down and right are reached by appropriate pairwise binding of lower level chains (as in $\mathbf{B}$, at $100 \mathrm{~ms}$ ). Diagonal directions involve the binding of three lower level chains (as in $\mathbf{B}$ at $300 \mathrm{~ms}$ ). The gray markers sample the trajectories at 25-ms intervals. lower level chains with which it is interconnected. The lower level chains have all-to-all cross-connections to allow all combinations of binding, but not all pairs of lower level chains can activate an upper level chain. Interconnections are realized such that each excitatory neuron in group $i$ of one chain has a small number of synapses to neurons in group $i+1$ of the other chain (Figure 2, black arrows). Additionally, each neuron in the network receives an independent Poisson spike train that yields a mean membrane potential of $15 \mathrm{mV}$ (Campbell's theorem, see Papoulis, 1991). To activate a lower level chain, a stimulus of a Gaussian distributed spike volley with the same parameters as a natural pulse propagating through the chain is applied to the initial group. The spike volley is characterized by the number of spikes contained $a$ and their temporal precision $\sigma$ (Diesmann et al., 1999). The model is summarized in Table 1. Unless otherwise stated, the model parameters are as given in Table 2 .

The activity in each upper level chain is mapped to a uniform velocity in one of the four directions "up," "down," "left," and "right," as described in Sec. 2.2. Note that this differs from the approach taken in Abeles et al. (2004), in which the lower level chains are considered as primitives and the upper level chains as composite objects. In this study the binding of lower level chains with an upper level chain constitutes a primitive, whereas concurrent activity in upper level chains represents a composite object. Figure $4 \mathrm{~B}$ demonstrates the binding of lower level chains to activate specific upper level chains. At $100 \mathrm{~ms}$, lower level chains 1 and 2 are stimulated near-synchronously (chain 1 at $100 \mathrm{~ms}$, chain 2 at $106 \mathrm{~ms}$ ). This causes them to bind and activate the upper level chain representing the direction "up." At around $300 \mathrm{~ms}$, chains 1,2, and 3 are stimulated near-synchronously (chain 1 at 300, chain 2 at 306, chain 3 at 302 ms). All three chains bind, thus activating the upper level chains representing the directions "up" and "right." As the number of bound chains increases, so does the level of inhibition, making it progressively harder for further chains to join the binding state. This can be seen at around $500 \mathrm{~ms}$, where although all lower level chains are stimulated (chain 1 at 500, chain 2 at 506, chain 3 at 502, chain 4 at $503 \mathrm{~ms}$ ), only chains 1, 3, and 4 enter a binding state, so only the upper level chains "left" and "down" are activated. Although the relative stimulation times for the first three chains are the same as before, the earlier stimulation of the fourth generates sufficient mutual inhibition such that chain 2 cannot join the binding state. The eight different drawing strokes that can be generated by the network through near-synchronous stimulation of two or three lower level chains (cf. Figure 4B at 100 and $300 \mathrm{~ms}$ ) are illustrated in Figure 4C.

\subsection{SEQUENTIAL COMPOSITION OF PRIMITIVES}

In the previous section we showed that by means of binding and competition, a hierarchic architecture of synfire chains can realize compositionality of behavior in terms of superimposing primitives. Here, we show that such an architecture is also capable of realizing sequential compositionality. In our example, this consists of generating a sequence of primitive and composed drawing strokes. We modify the model architecture illustrated in Figure $\mathbf{4 A}$ and summarized in Table 1 by connecting the lower level chains in a loop. In the modified architecture, the final group of each lower level chain projects to 40 neurons in the first groups of all the lower level chains. This can be visualized as a cylinder of interconnected synfire "rings" as shown in Figure 5A. All other aspects of the architecture, including the interconnections with the upper level chains, remain the same. Due to the cyclical structure, the activity in the network is self-sustained after a single initial stimulus (here, to chains 1 and 2 to activate the direction "up").Each "round" of activity results in a different binding among the lower level chains and thus to a random sequence of primitives and combinations of primitives. Figure $5 \mathrm{~B}$ shows a resulting motion trajectory from five seconds of network activity. The individual primitive 
Table 1 |Tabular description of network model after Nordlie et al. (2009).

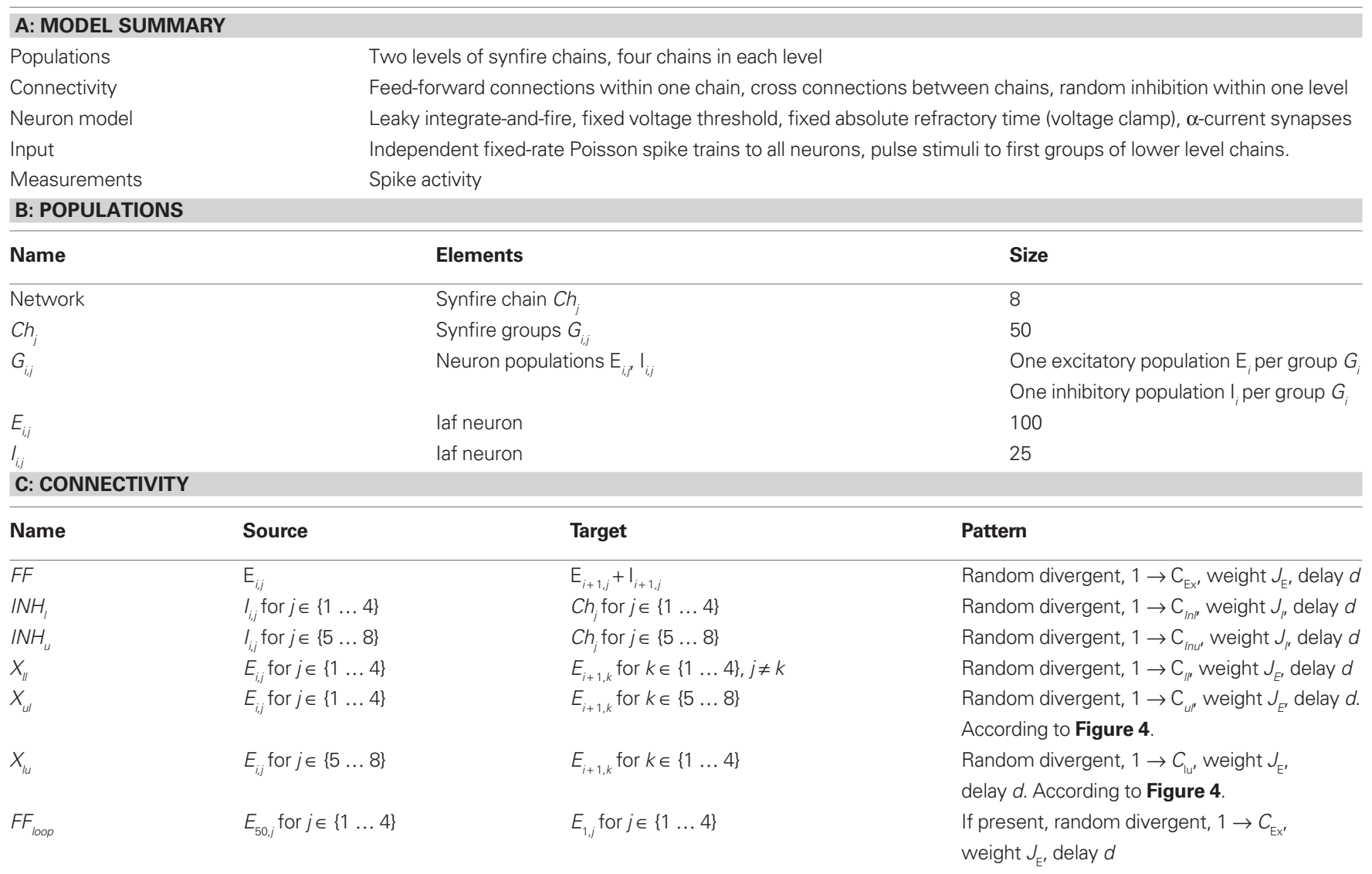

\section{D: NEURON MODELS}

\section{Name \\ Type \\ Subthreshold dynamics}

Spiking

\section{laf neuron}

Leaky integrate-and fire, $\alpha$-current input

if $\left(t>t^{*}+\tau_{\text {ref }}\right): d V / d t=-V / \tau_{\mathrm{m}}+I_{\text {syn }}(t) / C_{m}$ else: $V(t)=V_{\text {reset }}$

$I_{\text {syn }}=\sum_{i} \sum_{s \in S_{i}} J_{i} I_{\alpha}(t-s-d) \quad I_{\alpha}(t)=\frac{e}{\tau_{\alpha}} t e^{-t / \tau_{\alpha}}$

If $V(t-)<\Theta$ AND $V(t+) \geq \Theta$

1. set $t^{*}=t$

2. emit spike with time stamp $t^{*}$

\section{E: INPUT}

\begin{tabular}{ll}
\hline Type & Target \\
\hline Poisson generator & All $\mathrm{E}_{i^{\prime}} \mathrm{I}_{i}$ \\
Pulse packet & $\mathrm{E}_{1, j}+\mathrm{I}_{1, j}$ for $j \in\{1 \ldots 4\}$
\end{tabular}

\section{Description}

Independent for each neuron, rate $v_{x^{\prime}}$ weight $J_{x}$ Spike volleys with Gaussian distribution containing a spikes with temporal precision $\sigma$, weight $J_{p p}$

\section{F: MEASUREMENTS}

Spikes from all upper and lower level chains

and composed drawing strokes can be clearly seen, as can occasional sections where temporarily no meaningful binding state exists, resulting in somewhat more "dithery" drawing behavior.

The sequence of primitive and composed drawing strokes shown in Figure 5 is generated by a network with connections that close the lower level chains at the seam of the loop: the final group of each chain makes feed-forward connections to the initial groups of every chain. Due to this symmetry, any binding state is equally likely and a random sequence is produced. However, under the assumption that asymmetric connections between specific final and initial groups could develop, Figure 6 demonstrates that stereotypical or deterministic sequences can also be generated. For example, if chain 1 projects onto itself and chains 2 and 4 project onto each other, a sequence consisting of alternating "up" and "left" strokes is produced (Figure 6G). These results show that a hierarchic architecture of synfire chains is in principle capable of realizing at least a rudimentary syntax, as previously suggested (Bienenstock, 1991b). 
Table 2 | Simulation parameters.

\begin{tabular}{|c|c|c|}
\hline Name & Value & Description \\
\hline \multicolumn{3}{|c|}{ A: CONNECTIVITY } \\
\hline$C_{\mathrm{Ex}}$ & 40 & $\begin{array}{l}\text { Number of outgoing feed-forward } \\
\text { connections from each excitatory neuron }\end{array}$ \\
\hline$C_{|n|}$ & 5 & $\begin{array}{l}\text { Number of outgoing connections from } \\
\text { each lower level inhibitory neuron }\end{array}$ \\
\hline$C_{\text {Inu }}$ & 25 & $\begin{array}{l}\text { Number of outgoing connections from each } \\
\text { upper level inhibitory neuron }\end{array}$ \\
\hline$C_{\|}$ & 3 & $\begin{array}{l}\text { Number of outgoing lower level } \\
\text { cross-connections from each lower } \\
\text { level excitatory neuron }\end{array}$ \\
\hline$C_{\mathrm{ul}}$ & 8 & $\begin{array}{l}\text { Number of outgoing upper level } \\
\text { cross-connections from each lower } \\
\text { level excitatory neuron }\end{array}$ \\
\hline$C_{\text {lu }}$ & 8 & $\begin{array}{l}\text { Number of outgoing lower level } \\
\text { cross-connections from each upper } \\
\text { level excitatory neuron }\end{array}$ \\
\hline$J_{E}$ & $17.92 \mathrm{pA}$ & $\begin{array}{l}\text { Amplitude of excitatory connection, } \Rightarrow 0.2 \mathrm{mV} \\
\text { EPSP amplitude }\end{array}$ \\
\hline$J_{1}$ & $-71.70 \mathrm{pA}$ & $\begin{array}{l}\text { Amplitude of inhibitory connection, } \Rightarrow-0.8 \mathrm{mV} \\
\text { EPSP amplitude }\end{array}$ \\
\hline$d$ & $1 \mathrm{~ms}$ & Synaptic transmission delay \\
\hline \multicolumn{3}{|c|}{ B: NEURON MODEL } \\
\hline$\tau_{\mathrm{m}}$ & $20 \mathrm{~ms}$ & Membrane time constant \\
\hline$C_{m}$ & $200 \mathrm{pF}$ & Membrane capacitance \\
\hline$\Theta$ & $20 \mathrm{mV}$ & Fixed firing threshold \\
\hline$V_{0}$ & $0 \mathrm{mV}$ & Resting potential \\
\hline$V_{\text {reset }}$ & $V_{0}$ & Reset potential \\
\hline$\tau_{\text {ref }}$ & $2 \mathrm{~ms}$ & Absolute refractory period \\
\hline$\tau_{\alpha}$ & $1 \mathrm{~ms}$ & Rise time of post-synaptic current \\
\hline \multicolumn{3}{|c|}{ C: INPUT } \\
\hline$v_{x}$ & $6157 \mathrm{~Hz}$ & External Poisson rate \\
\hline$J_{x}$ & $8.96 \mathrm{pA}$ & $\begin{array}{l}\text { Amplitude of external excitatory } \\
\text { connection } \Rightarrow 0.1 \mathrm{mV} \text { EPSP amplitude }\end{array}$ \\
\hline$J_{p p}$ & $17.92 \mathrm{pA}$ & $\begin{array}{l}\text { Amplitude of pulse packet } \\
\text { connection } \Rightarrow 0.2 \mathrm{mV} \text { EPSP amplitude }\end{array}$ \\
\hline$a$ & 100 & Number of spikes in a volley \\
\hline$\sigma$ & $1 \mathrm{~ms}$ & Standard deviation of a Gaussian spike volley \\
\hline
\end{tabular}

\section{A}

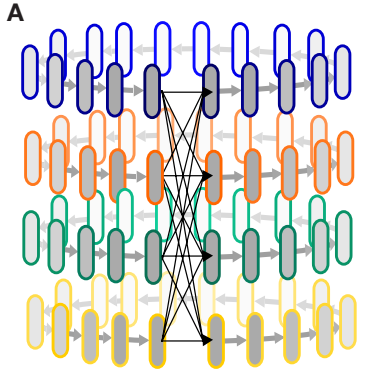

\section{B}

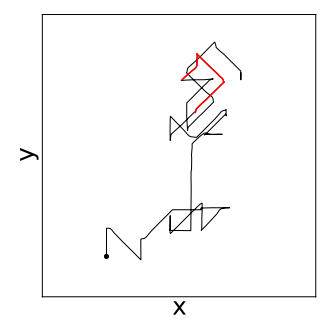

FIGURE 5 | Generation of random sequences. (A) Modified design of the lower level chains (1-4 of Figure 4). The system is looped by connecting the final groups of all the chains to all the first groups. The upper level system remains unchanged. (B) Trajectory generated by $5 \mathrm{~s}$ of network activity after an initial stimulus to chains 1 and 2 , leading to an initial up movement. The black dot marks the starting point. The red section of the trajectory is investigated further in Figure $\mathbf{8}$.
A

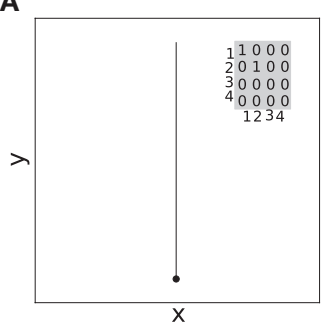

C

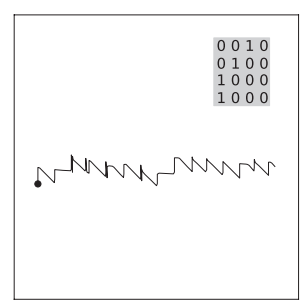

E

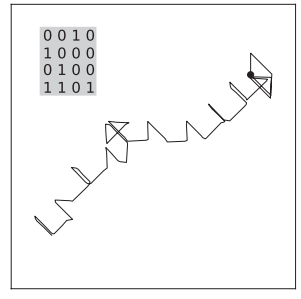

G

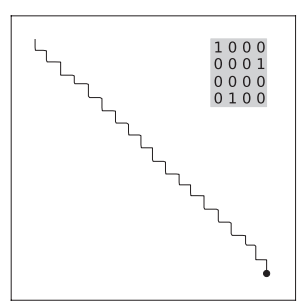

B

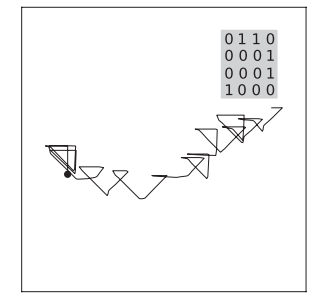

D

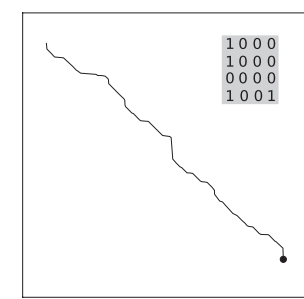

$\mathbf{F}$

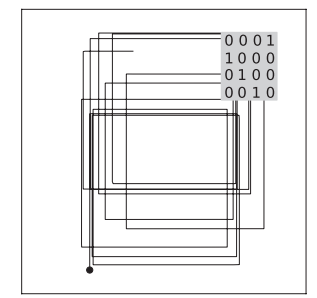

H

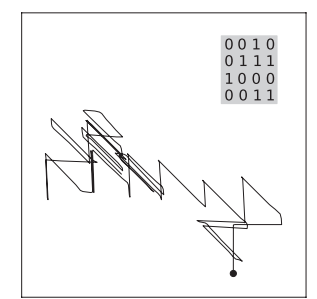

FIGURE 6 | Generation of non-random sequences. (A-H) Example trajectories generated by $5 \mathrm{~s}$ of network activity for networks with different connectivities between the final and initial groups of the lower level chains. Insets show the connectivity matrices: a 1 in row $i$, column $j$, indicates that the final group of chain $j$ is connected to the initial group of chain $i, 0$ indicates that there is no connection.

\subsection{LIMITS TO SIMULTANEITY AND SEQUENTIALITY}

Having demonstrated in the previous sections that an architecture of synfire chains can realize both simultaneous and sequential compositionality, we now investigate the robustness of this finding using the measures $p_{\text {sim }}$ and $p_{\text {seq }}$ defined in Sec. 2.3. The simultaneity measure $p_{\text {sim }}$ is the inverse of the average number of chains active in the upper level. Figure 7A shows $p_{\text {sim }}$ as a function the number of outgoing connections made by each upper and lower level inhibitory neuron. For strong upper level inhibition and weak lower level inhibition, $p_{\mathrm{sim}}=1$, indicating that only one chain is active at a time and thus that the criterion of simultaneity is not fulfilled. This is due to the stiff competition in the upper level preventing multiple 


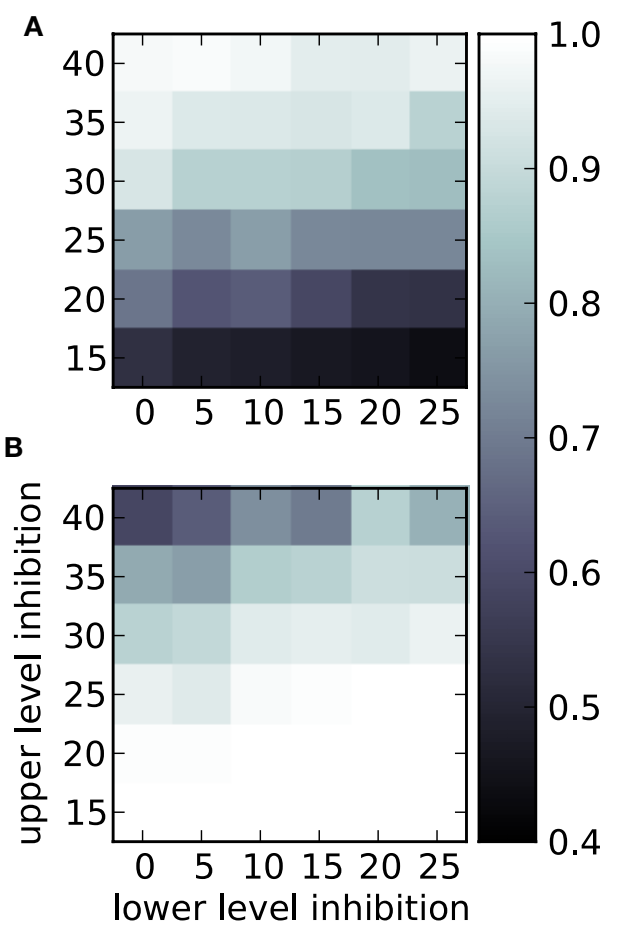

FIGURE 7 | Limits to simultaneity and sequentiality. (A) The simultaneity measure $p_{\text {sim }}$ applied to $20 \mathrm{~s}$ of network activity as a function of the number of outgoing connections made by each inhibitory neuron on the lower (horizontal axis) and upper (vertical axis) levels. (B) As in (A) but for the sequentiality measure $p_{\text {seq }}$.

(C) Trajectories generated by $5 \mathrm{~s}$ of network activity. Each trajectory is initialized from

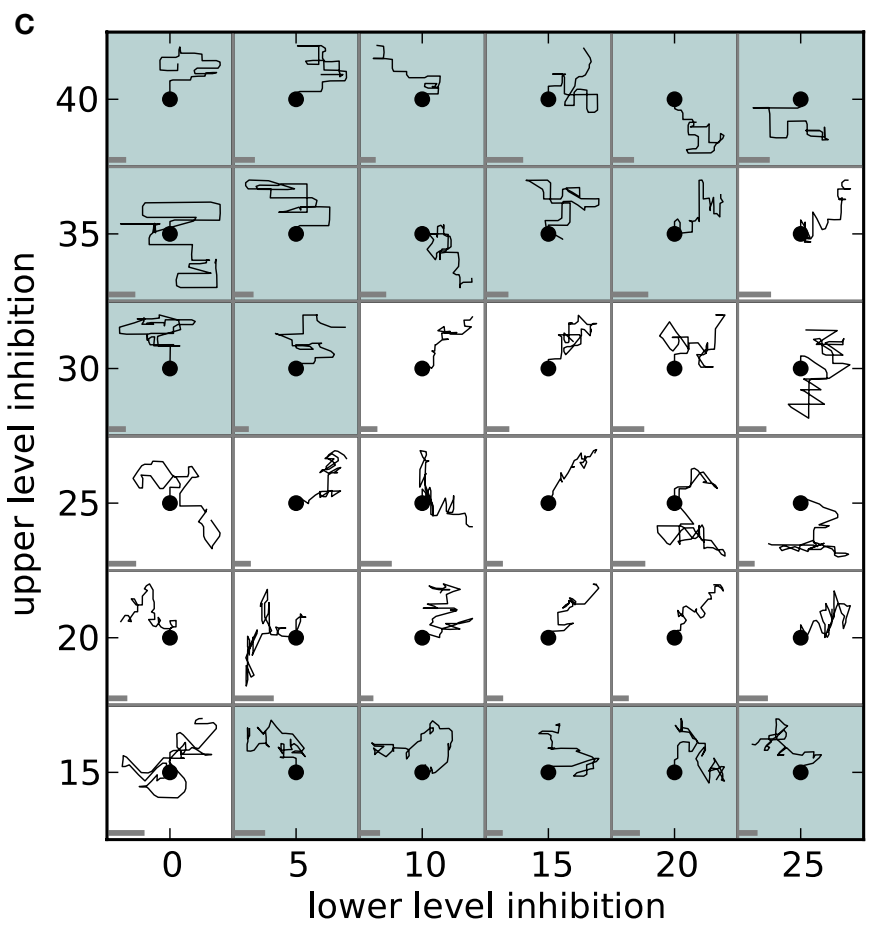

the point marked with the dot by an initial stimulus to chains 1 and 2 to activate the direction "up." Trajectories are scaled for visual clarity; to indicate the scaling, the gray bar in each cell shows the size of an arbitrary length unit. A white background color indicates that the network activity generating the trajectory meets both the criteria for simultaneity $\left(0.5<p_{\text {sim }}<0.9\right)$ and sequentiality $\left(p_{\text {seq }} \geq 0.9\right)$. upper level chains from entering a binding state. For weak upper level inhibition and strong lower level inhibition, $p_{\text {sim }}$ is reduced to 0.4 , indicating that on average more than two upper level chains are active. Such low values of $p_{\text {sim }}$ also violate simultaneity, as they can only occur in our simple model if incompatible primitives are being executed at the same time. We therefore define $0.5<p_{\text {sim }}<0.9$ as the range in which simultaneity is fulfilled.

The sequentiality measure $p_{\text {seq }}$ is the proportion of rounds of activity in the lower level for which at least one upper level chain was significantly active. For weak upper level inhibition, $p_{\text {seq }}=1$, indicating that there is always at least one upper level chain active and thus that the sequence of primitives is unbroken. For stronger upper level inhibition $p_{\text {seq }}$ drops to 0.67 . This means that the activity in the lower level chains fails to ignite activity in the upper level for one third of the rounds of activity. We define a successful sequential execution of primitives to have taken place when at least one upper level chain is active in at least $90 \%$ of all rounds, i.e., $p_{\text {seq }} \geq 0.9$.

We apply our criteria for simultaneity, $0.5<p_{\text {sim }}<0.9$, and sequentiality, $p_{\text {seq }} \geq 0.9$, in Figure 7C, which shows example sequences of drawing strokes generated by the model for different configurations of upper level and lower level inhibition. The white background demarcates the range of parameters for which our criteria for simultaneity and sequentiality are both met. In the top left section $p_{\text {seq }}$ is too low and $p_{\text {sim }}$ is too high, resulting in short sequences consisting only of individual primitives. In the bottom right section $p_{\text {sim }}$ is too low. The selection of incompatible primitives generates dithery trajectories. In the middle section both simultaneity and sequentiality are fulfilled. The sequences are long and contain both individual primitives and compatible combinations. Since previous investigation have revealed fairly general conditions for the robust propagation of activity in synfire chains (Herrmann et al., 1995; Diesmann et al., 1999, 2001), we conclude that the competition between primitives is determined by the balance of inhibition in the upper and lower levels, and that the criteria of simultaneity and sequentiality can be met for a wide range of parameters.

\subsection{ACTIVITY SIGNATURES OF SYNFIRE CHAIN COMPUTATION}

So far we have studied the complex behavior that can be generated out of superpositions and sequences of simple primitives, focusing on the behavioral output of our model. In this section, we will examine what features of the dynamics could be detectable by an experimenter investigating the nature of neural activity generating complex behavior. A researcher may measure mass signals of neural activity, such as the local field potential (LFP), the electroencephalogram (EEG), or the electrocorticogram (EcoG). Alternatively, the single unit activity of a limited number of neurons can be recorded. In the latter case, current techniques do not allow massively parallel recordings; through multiple electrodes or optical imaging methods, measurements would be unlikely to record the spike trains of more than 200 neurons. Of those neurons, it cannot be expected that all of them are involved with the generation of the behavior under investigation. Moreover, it cannot be assumed that the ordering of the spike trains in the recording reflects the ordering of connectivity in any underlying feed-forward structure. 
Spike activity recorded from our model therefore constitutes by far the best case in terms of analysis, as all neurons are only involved with the generation of the trajectory and the underlying structure is known.

Figure 8 shows the spike activity recorded for a short section of the drawing displayed in Figure 5. When the activity is displayed taking the architecture of the underlying synfire chains into consideration (Figure 5B), the relationship of the spiking activity to the generated behavior is clear. Due to the connectivity of all the final groups to all the initial groups of the lower level chains, the synfire activity in all the lower level groups is approximately synchronous. As the activities of the individual lower level chains are plotted over one another (cf. Figure 4), the collective activity appears similar to the activity in just one chain. Each time the activity volley reaches the end of the lower level chains, a new binding state is possible. The result is a different selection of upper level chains and thus a different drawing stroke or combination of drawing strokes (Figure 5A).

If the underlying structure of the network is not taken into consideration, an attribution of the generated behavior to the spiking activity becomes much harder. Figures 5C,D show the shuffled activity of 200 neurons from the upper and lower levels, respectively. The highly ordered spike activity of Figure 5A that is the typical hallmark of synfire activity is no longer visible. However, some signatures of synfire activity are still visible. The spike trains from the lower level chains (Figure 5C) exhibit a high degree of repetition, as the synfire activity repeats in every chain every $125 \mathrm{~ms}$. Repeating spike patterns have previously been identified as possible indications of synfire activity (e.g., Abeles et al., 1993). The spike trains from the upper level (Figure 5D) do not show such strong repetition, but a decrease in rate is clearly visible at the beginning of each trajectory segment. Even though the model dynamics consists solely of near-synchronous activity volleys, vertical stripes in the spike activity plots indicating strong synchrony only become evident when considering a much larger amount of neurons than could reasonably be recorded using currently available techniques (Figures 8B,D). We therefore conclude that signatures of synfire activity are unlikely to be found by searching for synchrony in spike trains.

As an alternative to massive parallel recordings of single unit activity, mass signals can be recorded to examine global effects. We have modeled such a signal as a histogram of the shuffled spiking data in Figures 8C-F. A particularly good impression of the global dynamics is gained when a large number of neurons contribute to the mass signal (Figures 5E,F). The large variance of the signal indicates a strong global synchronization, exhibiting fast ripples
A

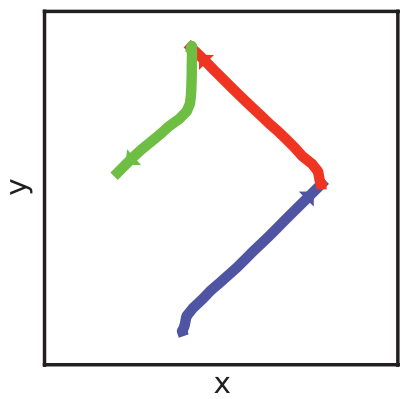

B

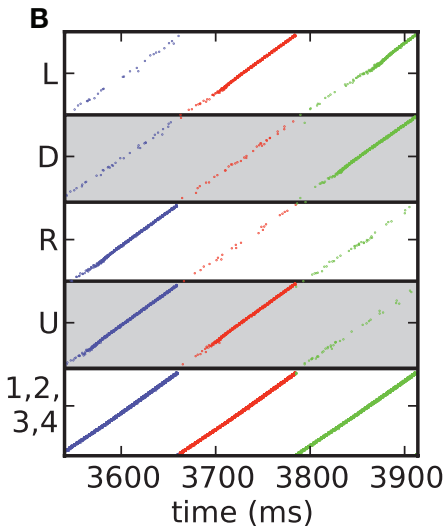

C

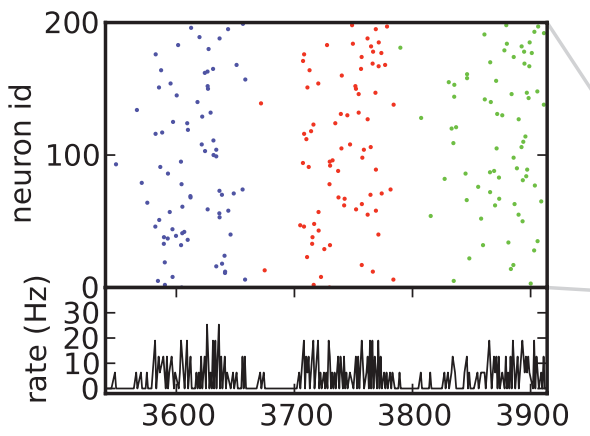

D

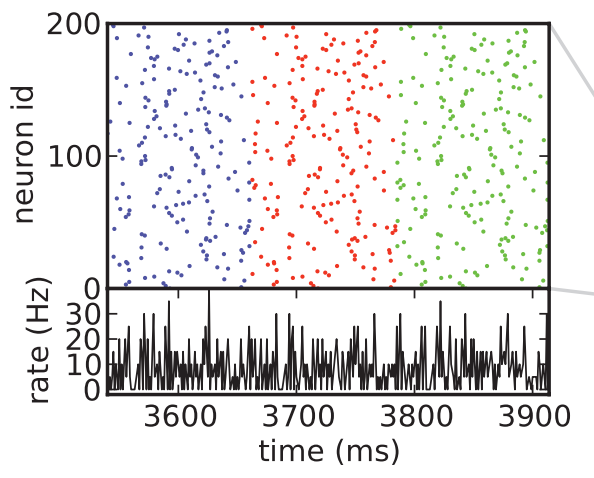

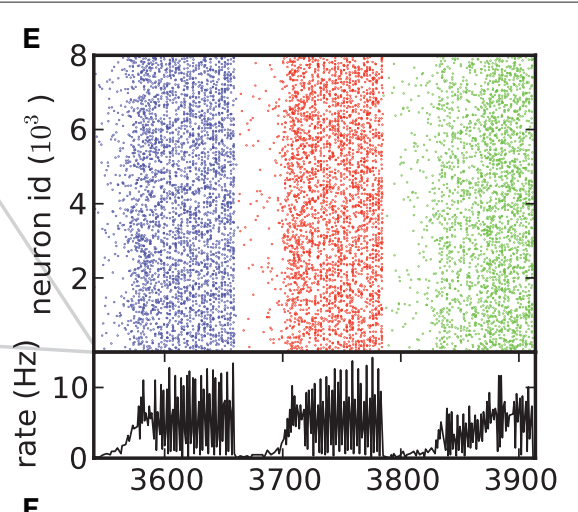

F

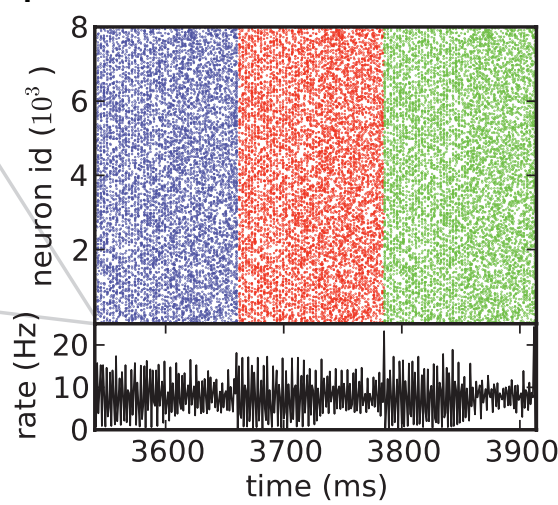

FIGURE 8 | Activity signatures during action execution. (A) Enlargement of the section of the trajectory marked in red in Figure 5B. The section contains three combinations of primitives. First, an "up/right" stroke (blue), then "up/left" (red), followed by a stroke which is initially "down" but turns into "left/down" midway through execution (green). Arrowheads mark the direction of stroke execution. (B) The underlying synfire chain activity. Each spike time marker is colored according to the drawing stroke in $(\mathbf{A})$ that is being generated at that time. (C) Upper panel: shuffled spiking activity of 200 neurons from the upper level chains, spike time markers colored as in (B). Lower panel: histogram of spiking activity with a bin size of $1 \mathrm{~ms}$ and normalized by the number of neurons. (D) As in (C), but for the lower level chains. (E,F) As in (C,D), but for 8000 neurons. Gray lines indicate the range of neurons shown in (C) and (D). 
at around $400 \mathrm{~Hz}$. The highly synchronous activity periods are interspersed with periods of silence (Figure 8E) or less synchronous activity (Figure 8F). These results enable us to make specific predictions about the relationship of an experimental protocol to the activity patterns observed in collective signals. First, execution of a primitive or a set of primitives is accompanied by high-frequency fluctuations in collective signals. Second, this high-frequency component is suppressed at decision points in the experimental protocol where a set of primitives is about to be activated.

\section{DISCUSSION}

In this study we demonstrate that a simple hierarchic architecture of synfire chains is able to generate composed behavior. To visualize the computational features of such a network, we associated primitive activity with drawing strokes in different directions. The network can combine orthogonal primitive drawing strokes into a composed diagonal stroke. In our model, the lower level chains have all-to-all cross-connections, so all specific combinations of chains can bind together. However, not all combinations of lower level chains activate upper level chains. This implements a semantics in the sense of assigning meaning to the binding states of lower level chains.

When the lower level chains are connected in a loop, the selfsustained activity in the network produces sequences of primitive and composed strokes. The characteristics of the sequences depend on the structure of the feed-forward connections between the final and initial groups of the lower level synfire chains. If these connections are symmetric, any sequence of primitives can occur, thus generating an random trajectory. By breaking the symmetry (see also Wennekers et al., 2006; Wennekers, 2007), it is possible to prescribe the transition probabilities between one primitive and the next, i.e., a basic syntax can also be implemented.

We have demonstrated that a single network model can generate actions that exhibit both simultaneity and sequentiality, resulting in much richer behavior than previous synfire chain based models which studied these aspects in isolation. Models accounting for sequentiality in the production of birdsong syntax (Okanoya, 2004; Jin, 2009) have not considered the superposition of primitives, as this does not occur in the target system. Similarly, studies demonstrating superposition (Abeles et al., 2004) did not provide an explanation for series of actions.

The two aspects of compositionality are realized by two different dynamical properties of synfire chains. Simultaneity is brought about by the synchronization of synfire chains, whereas sequentiality relies on feed-forward connections between the final group of one chain and the initial groups of potential successor chains. Hence we can derive the key architectural principles of a network model capable of combining both aspects of compositionality. Sequences of actions can be carried out if the final group of the synfire chain of one primitives makes feed-forward connections to the initial groups of all possible successor primitives, such that the activity volley can propagate from one chain to the next. The strength of the feed-forward connections determines the transition probabilities. Primitives that can be carried out simultaneously must be capable of mutual excitation to synchronize, whereas primitives that cannot be carried out simultaneously need mutual inhibition. The strength of the inhibition regulates the stiffness of the competition.
Using quantitative measures of sequentiality and simultaneity during synfire chain activity, we conclude that sequential and simultaneous compositionality is achievable over a wide range of balanced inhibition in the upper and lower levels. For simple models like the one investigated here, including inhibitory neurons in the synfire chains that make random connections to other neurons in the same level is sufficient; for more complicated models structured inhibition between specific groups of primitives might be necessary. Recently, first steps have been made toward the construction of large-scale compositional systems (Trengove, 2007).

The compatibility of the two types of synfire chain computation and the diversity of the behavior produced lend support to the hypothesis that synfire chains provide the substrate for some cortical functions. Specifically, our results support the theory that synfire chains are a possible substrate for the recognition and production of sequences, for example in processing language (Bienenstock, 1991a,b; Pulvermüller, 1999, 2002) or planning and executing motor programs (Jin, 2009; Hanuschkin et al., 2010b).

However, the two-dimensional drawing strokes we selected as primitives are merely an easy visualization of complex behavior, rather than attempting to account for the generation of movement. Although there is evidence for motor primitives and compositionality (Soechting and Terzuolo, 1987; Thoroughman and Shadmehr, 2000; Polyakov et al., 2009a,b), there are many features of motor behavior which cannot be captured by such a simple model as studied here. For example, a movement can be performed quickly or slowly, whereas a synfire chain is active for a fixed duration, in our model in the order of $100 \mathrm{~ms}$. This duration is in line with the generation of birdsong syllable sequences (Woolley and Rubel, 1997; Hahnloser et al., 2002; Sakata and Brainard, 2008), but appears too short to model elongated movements such as reaching, which are executed within about $500 \mathrm{~ms}$. Generally, longer or shorter activity durations can be generated by adjusting the chain length chains or the propagation speed through appropriately chosen synaptic delays or PSP rise-times (Wennekers and Palm, 1996). On the other hand, a single primitive does not necessarily need to represent a complete action. In the terminology of Rohrer et al. (2002), primitives represent the "atoms" of behavior. Any desired duration can be reached by appropriate concatenation.

Moreover, motor behavior is typically more complex than the straight lines at constant velocity used in this study for the purposes of illustration. To extend this model to movement compositionality would therefore require the identification of more realistic primitives. Candidates include dynamic motion primitives as force fields (Mussa-Ivaldi and Bizzi, 2000) and kinematic primitives such as parabolas (Flash and Handzel, 2007; Polyakov et al., 2009b). However, the use of more complex primitives immediately raises the question of how an appropriate coordinate system can be defined. The activity of prefrontal neurons in reaching tasks has been shown to represent coordinate systems centered around the hand, target, eye fixation point, and even the shoulder (Pesaran et al., 2006). When a complex movement is performed, like handwriting or grasping an object, basic motion features can be observed which repeat elsewhere in an altered fashion. For example, we can almost immediately write using our foot in the sand, without having to practice it. This suggests 
that the writing trajectories of letters have not been trained to be restricted to our hand but as a sequence of accelerations in general. The representation of trajectories may even be independent of a particular task (such as writing or catching a ball), but rather an abstract instance to be used in a variety of contexts. This leads to the conclusion that complex movements may be assembled from simpler, context-free building blocks. Hierarchical organizations of synfire chains might therefore realize a movement syntax within one coordinate system or across multiple systems. This could enable a form of binding-based coordinate transformation (Salinas and Abbott, 1995).

The existence of synfire chains and the capacity of the brain to develop them are aspects which have been presupposed in this work. Synfire chains have been postulated to underlie experimentally observed precise spike timing, for example in mammalian cortex (Eckhorn et al., 1988; Abeles et al., 1993; Prut et al., 1998; Ikegaya et al., 2004; Pulvermüller and Shtyrov, 2009) or avian area HVC (Hahnloser et al., 2002; Jin et al., 2007; Kozhevnikov and Fee, 2007) and methods are being developed to detect them in parallel spike train recordings (Schrader et al., 2008). Though it might be possible that such feed-forward structures are hardwired in the cortical or basal ganglia structure, it appears more plausible that they are imprinted by some Hebbian-like mechanism. For this, neuron groups must be repeatedly activated, possibly by means of sensory input or from other areas such as the hippocampus (Ji and Wilson, 2007), leading to stronger synapses between groups. Studies on this issue report conflicting results. While feed-forward structure has been shown to develop in some network models (Izhikevich et al., 2004; Buonomano, 2005; Doursat and Bienenstock, 2006; Jun and Jin, 2007; Fiete et al., 2010), these results do not scale up to large-scale networks with biologically realistic numbers of synapses per neuron (Morrison et al., 2007; Kunkel et al., 2010). Further studies are needed to determine the conditions under which the divergent-convergent connectivity of synfire chains can develop in cortical networks.

Despite these issues, the indirect evidence for synfire chains is great enough that one can legitimately be interested in investigating their capabilities. Indeed, although our model consists exclusively of synfire chains, standard measurement techniques applied to the spiking data can also only produce indirect evidence for synfire activity. Only the activity of the lower level neurons exhibited strong patterns; upper level activity indicated the transition from one primitive or group of primitives to the next with a decrease in spiking activity. By construction, our model predicts a synchronized activity between neurons that code for a particular primitive, as well as synchronization between the upper and lower levels. In principle, those levels may be located in different brain areas. Experimental studies have reported coherent oscillations between different motor areas during action execution, such as M1 and premotor areas (Brovelli et al., 2004).

The exact frequency of the oscillations visible in the mass signal during execution of a complex action is largely determined by the choice of synaptic delay; however, any selection within a reasonable range for this delay would result in oscillatory activity in the order of $100 \mathrm{~Hz}$. Induced gamma activity (as opposed to evoked gamma) lies well in this order (Ball et al., 2008) and may reflect such activity. In addition, our model predicts the presence of desynchronized activity at the transition between primitives, thus potentially accounting for event-related desynchronization (Pfurtscheller and Lopes da Silva, 1999; Miller et al., 2010).

Our model thus provides us with a theoretical basis to investigate the correlation between composed behavior and neuronal activity. For example, the activity of the network is interpreted in terms of a rate code of directionally tuned neuronal populations. However, as each neuron is only briefly active during the propagation of activity through a synfire chain, it only contributes to a particular phase of a drawing stroke, rather than to the entire duration. Additionally, as the lower level chains are constantly active in the looped network, they can be active during a drawing stroke in any direction. Hence, although the entire system produces reliable output, many of the neurons exhibit weak or negligible direction tuning. This insight may well be relevant for experimental studies of the neural correlates of motor control, which classically assume that neurons involved with movement exhibit directional tuning.

To execute specific non-ongoing sequences, a correct sequence of synchronous pulse stimuli would need to be delivered to the first groups of the relevant lower level chains whilst other activity is inhibited. Up to now, no attempt has been made to explain where the precise instruction pulse sequences arriving at the lower level system should originate. Due to the relatively small binding window $(<20 \mathrm{~ms})$, stimuli must be generated very precisely and reliably. Though sensory systems might be too slow to reach such a precision (Thorpe et al., 1996), higher areas such as the cerebellum or the basal ganglia are able to produce this kind of time locking (Dreher and Grafman, 2002). In this work, our focus lies on the generation of the sequences; learning the correct sequence of stimuli to produce a useful sequence of primitives is outside the current scope. However, other studies have already made significant progress in developing learning agents that would be able to learn such sequences (e.g., Baras and Meir, 2007; Farries and Fairhall, 2007; Izhikevich, 2007; Potjans et al., 2009a,b; Vasilaki et al., 2009). Therefore, an interesting future extension of our work is to investigate to what extent our model can fulfill the role of an action selector in network models of learning agents.

Due to the mutual inhibition between chains (unbound chains are less likely to join a synchronized state), our system implements a winner-take-all functionality of action selection. Competing synfire chains provide a neural solution to the selection problem, and exhibit many features of an ideal selection mechanism (for a review see Redgrave et al., 1999). An effective action selector has to choose from a large pool of behaviors depending on their importance or feasibility. Selection must be carried out quickly and also under close-run conditions (clean switching), a criterion which is met in our model. During execution, competing "votes" may not interfere with the current behavior (distortion), a property also inherent in the running of synfire chains. Once a primitive has been activated, it is executed completely, i.e., actions have persistence. Conversely, in any trajectory involving a sequence of primitives, there is an opportunity to choose a more important action at the switch-over point between primitives, i.e., the system exhibits interruptibility. In future work we will investigate the relationship between the structure and dynamics of the hierarchic model and its expression of these features. 


\section{ACKNOWLEDGMENTS}

This work is partially supported by DIP F1.2, EU Grant 15879 (FACETS), BMBF Grant 01GQ0420 to BCCN Freiburg, Helmholtz Alliance on Systems Biology (Germany), Next-Generation Supercomputer Project of MEXT (Japan), Neurex, and the Junior Professor Program of Baden-Württemberg. We acknowledge fruitful discussion with the DIP collaboration, in particular M. Abeles, T. Flash, M. Herrmann, and F. Polyakov. We are thankful to M. Herrmann and A. Hanuschkin for their constructive criticism of an earlier version of this manuscript. Sven Schrader is most grateful to T. Flash for the scientific stay he enjoyed in May 2007

\section{REFERENCES}

Abeles, M. (1982). Local Cortical Circuits: An Electrophysiological Study. Studies of Brain Function. Berlin: SpringerVerlag.

Abeles, M. (1991). Corticonics: Neural Circuits of the Cerebral Cortex (1st Edn.). Cambridge: Cambridge University Press.

Abeles, M., Bergman, H., Margalit, E., and Vaadia, E. (1993). Spatiotemporal firing patterns in the frontal cortex of behaving monkeys. J. Neurophysiol. $70,1629-1638$.

Abeles, M., and Gat, I. (2001). Detecting precise firing sequences in experimental data. J. Neurosci. Methods 107, 141-154.

Abeles, M., Hayon, G., and Lehmann, D. (2004). Modeling compositionality by dynamic binding of synfire chains. J. Comput. Neurosci. 17, 179-201.

Anderson, J. C., and Martin, K. A. C. (2009). The synaptic connections between cortical areas V1 and V2 in macaque monkey. J. Neurosci. 29, $11283-11293$.

Arnoldi, H. M., and Brauer, W. (1996). Synchronization without oscillatory neurons. Biol. Cybern. 74, 209-223.

Baker, S. N., and Lemon, R. (2000). Precise spatiotemporal repeating patterns in monkey primary and supplementary motor areas occur at chance level. J. Neurophysiol. 84, 1770-1780.

Baker, S. N., Spinks, R., Jackson, A., and Lemon, R. N. (2001). Synchronization in monkey motor cortex during a precision grip task. I. Task-dependent modulation in single-unit synchrony. J. Neurophysiol. 85, 869-885.

Ball, T., Demandt, E., Mutschler, I., Neitzel, E., Mehring, C., Vogt, K., Aertsen, A., and Schulze-Bonhage, A. (2008). Movement related activity in the high gamma range of the human EEG. Neuroimage 41, 302-310.

Baras, D., and Meir, R. (2007). Reinforcement learning, spiketime-dependent plasticity, and the BCM rule. Neural Comput. 19, 2245-2279.

Bienenstock, E. (1991a). "Notes on the growth of a 'composition machine," in Proceedings of the Royaumont Interdisciplinary Workshop on Compositionality in Cognition and Neural Models, eds D. Andler, E. Bienenstock, and B. Laks (Oise,
France: Abbaye de Royaumont, Asniere s), 1-19.

Bienenstock, E. (1991b). "Suggestions for a neurobiological approach to syntax," in Proceedings of the Royaumont Interdisciplinary Workshop on Compositionality in Cognition and Neural Networks - II, eds D. Andler, E. Bienenstock, and B. Laks (Oise, France: Abbaye de Royaumont, Asniere s.), 13-21.

Bienenstock, E. (1995). A model of neocortex. Netw. Comput. Neural Syst. 6, 179-224.

Brovelli, A., Ding, M., Ledberg, A., Chen, Y., Nakamura, R., and Bressler, S. L. (2004). Beta oscillations in a largescale sensorimotor cortical network: directional influences revealed by Granger causality. Proc. Natl. Acad. Sci. U.S.A. 101, 9849-9854.

Buonomano, D.V.(2005). A learning rule for the emergence of stable dynamics and timing in recurrent networks. $J$. Neurophysiol. 94, 2275-2283.

Date, A., Bienenstock, E., and Geman, S. (1999). A statistical technique for detection of fine temporal structure in multi neuronal spike trains. Soc. Neurosci. Abstr. 25, 1441.

Diesmann, M., Gewaltig, M.-O., and Aertsen, A. (1999). Stable propagation of synchronous spiking in cortical neural networks. Nature 402, 529-533.

Diesmann, M., Gewaltig, M.-O., Rotter, S., and Aertsen, A. (2001). State space analysis of synchronous spiking in cortical neural networks. Neurocomputing 38-40, 565-571.

Douglas, R. J., and Martin, K. A. (2007) Recurrent neuronal circuits in the neocortex. Curr. Biol. 17, 496-500.

Doursat, R., and Bienenstock, E. (2006). "The self-organized growth of synfire patterns," in 10th International Conference on Cognitive and Neural Systems (ICCNS), Massachusetts: Boston University.

Dreher, J. C., and Grafman, J. (2002). The roles of the cerebellum and basa ganglia in timing and error prediction. Eur. J. Neurosci. 16, 1609-1619.

Eckhorn, R., Bauer, R., Jordan, W., Brosch, M., Kruse, W., Munk, M., and Reitböck, H. J. (1988). Coherent oscillations: a mechanism of feature linking in the visual cortex? Biol. Cybern. 60, 121-130.
Farries, M. A., and Fairhall, A. L. (2007). Reinforcement learning with modulated spike timing-dependent synaptic plasticity. J. Neurophysiol. 98 3648-3665.

Fiete, I. R., Senn, W., Wang, C. Z. H., and Hahnloser, R. H. R. (2010). Spiketime-dependent plasticity and heterosynaptic competition organize networks to produce long scale-free sequences of neural activity. Neuron 65, 563-576.

Flash, T., and Handzel, A. A. (2007) Affine differential geometry analysis of human arm movements. Biol. Cybern. 96, 577-601.

Georgopoulos, A., Kalaska, J., Caminiti, R., and Massey, J. T. (1982). On the relations between the direction of twodimensional arm movements and cell discharge in primate motor cortex. $J$. Neurosci. 11, 1527-1537.

Gerstein, G. (2004). Searching for significance in spatio-temporal firing patterns. Acta Neurobiol. Exp. 2 203-207.

Gewaltig, M.-O., and Diesmann, M. (2007). NEST (neural simulation tool). Scholarpedia 2, 1430

Gewaltig, M.-O., Diesmann, M., and Aertsen, A. (2001). Propagation of cortical synfire activity: survival probability in single trials and stability in the mean. Neural Netw. 14, 657-673.

Gray, C. M., and Singer, W. (1989). Stimulus-specific neuronal oscillations in orientation columns of cat visual cortex. Proc. Natl. Acad. Sci. U.S.A. 86:1698-1702.

Hahnloser, R. H., Kozhevnikov, A. A., and Fee, M.S. (2002). An ultra-sparse code underlies the generation of neural sequences in a songbird. Nature 419 65-70.

Hanuschkin, A., Diesmann, M., and Morrison, A. (2010a). A reafferent model of song syntax generation in the Bengalese finch. BMC Neurosci. 11(Suppl. 1), P33. doi: 10.1186/1471 2202-11-S1-P33.

Hanuschkin, A., Herrmann, J. M. Morrison, A., and Diesmann, $M$ (2010b). Compositionality of arm movements can be realized by propagating synchrony. J. Comput. Neurosci. doi: 10.1007/s10827-010-0285-9. [Epub ahead of print]

Hasenstaub, A., Shu, Y., Haider, B. Kraushaar, U., Duque, A., and
McCormick, D. (2005). Inhibitory postsynaptic potentials carry synchronized frequency information in active cortical networks. Neuron 3 , 423-435.

Hayon, G., Abeles, M., and Lehmann, D. (2005). A model for representing the dynamics of a system of synfire chains. J. Comput. Neurosci. 18, 41-53.

Herrmann, M., Hertz, J. A., and PrügelBennett, A. (1995). Analysis of synfire chains. Network 6, 403-414.

Ikegaya, Y., Aaron, G., Cossart, R., Aronov, D., Lampl, I., Ferster, D., and Yuste, R. (2004). Synfire chains and cortical songs: temporal modules of cortical activity. Science 5670, 559-564.

Ikegaya, Y., Matsumoto, W., Chiou, H. Y., Yuste, R., and Aaron, G. (2008) Statistical significance of precisely repeated intracellular synaptic patterns. PLoS One 3:e3983. doi: 10.1371/ journal.pone.0003983.

Izhikevich, E. M. (2007). Solving the distal reward problem through linkage of STDP and dopamine signaling. Cereb. Cortex 17, 2443-2452.

Izhikevich, E. M., Gally, J.A., and Edelman, G. M. (2004). Spike-timing dynamics of neuronal groups. Cereb. Cortex 14, 933-944.

Jack, J. J. B., Noble, D., and Tsien, R. W. (1983). Electric Current Flow in Excitable Cells. Oxford: Oxford University Press.

Ji, D., and Wilson, M. A. (2007) Coordinated memory replay in the visual cortex and hippocampus during sleep. Nat. Neurosci. 10, 100-107. doi: $10.1038 / \mathrm{nn} 1825$.

Jin, D. Z. (2009). Generating variable birdsong syllable sequences with branching chain networks in avian premotor nucleus HVC. Phys. Rev. E. 80, 051902.

Jin, D. Z., Ramazanoglu, F. M., and Seung, H. S. (2007). Intrinsic bursting enhances the robustness of a neural network model of sequence generation by avian brain area HVC. J. Comput. Neurosci. 23, 283-299.

Jun, J. K., and Jin, D. Z. (2007). Development of neural circuitry for precise temporal sequences through spontaneous activity, axon remodeling, and synaptic plasticity. PLoS One 2(8):e723. doi: 10.1371/journal. pone. 0000723

Kohn, A., and Smith, M. A. (2005). Stimulus dependence of neuronal 
correlations in primary visual cortex of the Macaque. J. Neurosci. 25, 3661-3673.

Kozhevnikov, A., and Fee, M. S. (2007). Singing-related activity of identified HVC neurons in the zebra finch. $J$. Neurophysiol. 97, 4271-4283.

Kunkel, S., Diesmann, M., and Morrison, A. (2010). Limits to the development of feed-forward structures in large recurrent neuronal networks. Front. Comput. Neurosci. 4:160. doi: 10.3389/ fncom. 2010.00160

Li, M., and Greenside, H. (2006). Stable propagation of a burst through a onedimensional homogeneous excitatory chain model of songbird nucleus HVC. Phys. Rev. E. 74, 011918.

Long, M. A., Jin, D. Z., and Fee, M. S. (2010). Support for a synaptic chain model of neuronal sequence generation. Nature 18, 394-399.

Masuda, N. (2006). Simultaneous ratesynchrony codes in population of spiking neurons. Neural Comput. 18, 45-59.

Matsumura, M., Chen, D., Sawaguchi, T., Kubota, K., and Fetz, E. E. (1996). Synaptic interactions between primate precentral cortex neurons revealed by spike-triggered averaging of intracellular membrane potentials in vivo. J. Neurosci. 16, 7757-7767.

Miller, K. J., Schalk, .G, Fetz, E. E., den Nijs, M., Ojemann, J. G., and Rao, R.P. (2010). Cortical activity during motor execution, motor imagery, and imagery-based online feedback. Proc. Natl. Acad. Sci. U.S.A. 107, 4430-4435.

Mokeichev, A., Okun, M., Barak, O. Katz, Y., Ben-Shahar, O., and Lampl, I. (2007). Stochastic emergence of repeating cortical motifs in spontaneous membrane potential fluctuations in vivo. Neuron 53, 413-425.

Morrison, A., Aertsen, A., and Diesmann, M. (2007). Spike-timing dependent plasticity in balanced random networks. Neural Comput. 19, 1437-1467.

Morrison, A., Mehring, C., Geisel, T., Aertsen, A., and Diesmann, M. (2005). Advancing the boundaries of high connectivity network simulation with distributed computing. Neural Comput. 17, 1776-1801.

Mussa-Ivaldi, F. A., and Bizzi, E. (2000). Motor learning through the combination of primitives. Philos. Trans. R. Soc. Lond., B. 355, 1755-1769.

Mussa-Ivaldi, F. A., Giszter, S., and Bizzi, E. (1994). Linear combinations of primitives in vertebrate motor control. Proc. Natl. Acad. Sci. U.S.A. 91, 7534-7538.

Nordlie, E., Gewaltig, M.-O., and Plesser, H. E. (2009). Towards reproducible descriptions of neuronal network models. PLoS Comput. Biol. 5:e1000456, doi: 10.1371/journal. pcbi. 1000456

Okanoya, K. (2004). The Bengalese finch: a window on the behavioral neurobiology of birdsong syntax. Ann. N. Y. Acad. Sci. 1016, 724-735.

Oram, M.W., Wiener, M. C., Lestienne, R. and Richmond, B. J. (1999). Stochastic nature of precisely timed spike patterns in visual system neuronal responses. $J$. Neurophysiol. 81, 3021-3033.

Papoulis, A. (1991). Probability, Random Variables, and Stochastic Processes (3rd Edn.). Boston, MA: McGraw-Hill.

Pesaran, B., Nelson, M. J., and Andersen, R. A. (2006). Dorsal premotor neurons encode the relative position of the hand, eye, and goal during reach planning. Neuron 51, 125-134.

Pfurtscheller, G., and Lopes da Silva, F. H. (1999). Event-related EEG/MEG synchronization and desynchronization: basic principles. Clin. Neurophysiol. 110, 1842-1857.

Plamondon, R. (1995). A kinematic theory of rapid human movements. part I. movement representation and generation. Biol. Cybern. 72, 295-307.

Plesser, H. E., and Diesmann, M. (2009). Simplicity and efficiency of integrateand-fire neuron models. Neural Comput. 21, 353-359.

Polyakov, F., Drori, R., Ben-Shaul,Y.,Abeles, M., and Flash, T. (2009a). A compact representation of drawing movements with sequences of parabolic primitives. PLoS Comput. Biol. 5: e1000427. doi: 10.1371/journal.pcbi.1000427.

Polyakov, F., Stark, E., Drori, R., Abeles, M. and Flash, T. (2009b). Parabolic movement primitives and cortical states: merging optimality with geometric invariance. Biol. Cybern. 100, 159-184.

Potjans, W., Morrison, A., and Diesmann, M. (2009a). A spiking neural network model of an actor-critic learning agent. Neural Comput. 21, 301-339.

Potjans, W., Morrison, A., and Diesmann, M. (2009b). A spiking temporaldifference learning model based on dopamine-modulated plasticity. BMC Neurosci. 10(Suppl. 1), 140. doi: 10.1186/1471-2202-10-S1-P140.

Prut, Y., Vaadia, E., Bergman, H., Haalman, I., Hamutal, S., and Abeles, M. (1998). Spatiotemporal structure of cortical activity: properties and behavioral relevance. J. Neurophysiol. 79, 2857-2874.

Pulvermüller, F. (1999). Words in the brain's language. Behav. Brain Sci. 22, 253-279.

Pulvermüller, F. (2002). The Neuroscience of Language: On Brain Circuits of Words and Serial Order. Cambridge: Cambridge University Press.

Pulvermüller, F., and Shtyrov, Y. (2009). Spatiotemporal signatures of large-scale synfire chains for speech processing as revealed by MEG. Cereb. Cortex. 19, 79-88.

Redgrave, P., Prescott, T., and Gurney, K. (1999). The basal ganglia: a vertebrate solution to the selection problem? Neuroscience 89, 1009-1023.

Rohrer, B., Fasoli, S., Krebs, H. I., Hughes, R., Volpe, B., Frontera, W. R., Stein. J., and Hogan, N. (2002). Movement smoothness changes during stroke recovery. J. Neurosci. 22, 8297-8304.

Rotter, S., and Diesmann, M. (1999). Exact digital simulation of time-invariant linear systems with applications to neuronal modeling. Biol. Cybern. 81(5/6), 381-402.

Sakata, J. T., and Brainard, M. S. (2008) Online contributions of auditory feedback to neural activity in avian song control circuitry. J. Neurosci. 28 11378-11390.

Salinas, E., and Abbott, L. F. (1995) Transfer of coded information from sensory to motor networks. J. Neurosci. $15,6461-6474$.

Schrader, S., Grün, S., Diesmann, M., and Gerstein, G. (2008). Detecting synfire chain activity using massively parallel spike train recording. J. Neurophysiol. 100, 2165-2176.

Schrader, S., Morrison, A., and Diesmann, M. (2007). "A composition machine for complex movements," in Proceedings of the 31st Göttingen Neurobiology Conference, Göttingen, Germany, TS18-1C.

Shmiel, T., Drori, R., Shmiel, O., BenShaul, Y., Nadasdy, Z., Shemesh, M. Teicher, M., and Abeles, M. (2006). Temporally precise cortical firing patterns are associated with distinct action segments. J. Neurophysiol. 96 2645-2652.

Shmiel, T., Drori, R., Shmiel, O., BenShaul, Y., Nadasdy, Z., Shemesh, M. Teicher, M., Abeles, M., and Bass, H. (2005). Neurons of the cerebra cortex exhibit precise interspike timing in correspondence to behavior. Proc. Natl. Acad. Sci. U.S.A. 102, 18655-18657.

Soechting, J., and Terzuolo, C. (1987) Organization of arm movements. Motion is segmented. Neuroscience 23, 39-51.

Sosnik, R., Hauptmann, B., Karni, A., and Flash, T. (2004). When practice leads to co-articulation: the evolution of geometrically defined movement primitives. Exp. Brain Res. 156, 422-438.

Tanaka, K. (2003). Columns for complex visual object features in the inferotemporal cortex: clustering of cells with similar but slightly different stimulus selectivities. Cereb. Cortex 13, 90-99.

Thoroughman, K. A., and Shadmehr, R. (2000). Learning of action through adaptive combination of motor primitives. Nature 407, 742-747.

Thorpe, S., Fize, D., and Marlot, C. (1996). Speed of processing in the human visual system. Nature 381, 520-522.

Trengove, C. (2007). Storage capacity of a superposition of synfire chains using conductance-based integrate-and-fire neurons. BMC Neurosci. 8(Suppl. 2), P67. doi: 10.1186/1471-2202-8-S2P67.

Tuckwell, H. C. (1988). “The Lapique model of the nerve cell, chapter 3," in Introduction to Theoretical Neurobiology, Vol. 1, eds C. Cannings, F. C. Hoppensteadt, and L. A. Segel (Cambridge: Cambridge University Press), 85-123.

Vasilaki, E., Frémaux, N., Urbanczik, R., Senn, W., and Gerstner, W. (2009). Spike-based reinforcement learning in continuous state and action space: when policy gradient methods fail. PLoS Comput. Biol. 5: e1000586. doi: 10.1371/journal.pcbi.1000586

Wennekers, T. (2007). A cell assembly model for complex behaviour. Neurocomputation 70, 1988-1992.

Wennekers, T., Garagnani, M., and Pulvermüller, F. (2006). Language models based on Hebbian cell assemblies. J. Physiol. Paris 100, 16-30.

Wennekers, T., and Palm, G. (1996). "Controlling the speed of synfire chains," in Artificial Neural Networks - ICANN 96, eds C. von der Malsburg, W. von Seelen, J. C. Vorbrüggen, and B. Sendhoff (Berlin: Springer-Verlag), 451-456.

Woolley, S. M., and Rubel, E. W. (1997). Bengalese finches Lonchura Striata Domestica depend upon auditory feedback for the maintenance of adult song. J. Neurosci. 17, 6380-6390.

Conflict of Interest Statement: The authors declare that the research was conducted in the absence of any commercial or financial relationships that could be construed as a potential conflict of interest.

Received: 26 March 2010; accepted: 05 December 2010; published online: 05 January 2011

Citation: Schrader S, Diesmann $M$ and Morrison A (2011) A compositionality machine realized by a hierarchicarchitecture of synfire chains. Front. Comput. Neurosci. 4:154. doi: 10.3389/fncom.2010.00154 Copyright (c) 2011 Schrader, Diesmann and Morrison. This is an open-access article subject to an exclusive license agreement between the authors and the Frontiers Research Foundation, which permits unrestricted use, distribution, and reproduction in any medium, provided the original authors and source are credited. 